\title{
MiR-30 Family Potentially Targeting PI3K-SIAH2 Predicted Interaction Network Represents a Novel Putative Theranostic Panel in Non-small Cell Lung Cancer
}

\author{
Lawrence W. C. Chan ${ }^{1 *}$, Fengfeng Wang ${ }^{1}$, Fei Meng ${ }^{1}$, Lili Wang ${ }^{1}$, \\ Sze Chuen Cesar Wong ${ }^{1}$, Joseph S. K. Au ${ }^{2}$, Sijun Yang ${ }^{3}$ and William C. S. Cho ${ }^{4}$ \\ 1 Department of Health Technology and Informatics, Hong Kong Polytechnic University, Hong Kong, Hong Kong, \\ ${ }^{2}$ Hong Kong Adventist Hospital, Hong Kong, Hong Kong, ${ }^{3}$ ABSL-3 Laboratory at the Center for Animal Experiment and \\ State Key Laboratory of Virology, School of Medicine, Institute of Animal Model for Human Diseases, Wuhan University, \\ Wuhan, China, ${ }^{4}$ Department of Clinical Oncology, Queen Elizabeth Hospital, Hong Kong, Hong Kong
}

Non-small cell lung cancer (NSCLC) comprises about $84 \%$ of all lung cancers. Many treatment options are available but the survival rate is still very low due to drug resistance. It has been found that phosphoinositide-3-kinase (PI3K) affects sensitivity to tyrosine kinase inhibitors (TKIs), including gefitinib and erlotinib. Expression level of seven in absentia homolog 2 (SIAH2), an E3 ubiquitin-protein ligase, is upregulated in NSCLC and correlated with tumor grade. However, the relationship between PI3K and $\mathrm{SIAH} 2$ remains unclear and therefore it is not known whether they can act as treatment co-targets and theranostic dual markers for overcoming TKI resistance. It is worthy to note that $\mathrm{PI} 3 \mathrm{~K}$ and $\mathrm{SIAH} 2$ are potentially regulated by a common group of microRNAs in miR-30 family. Our bioinformatics analyses showed upregulated SIAH2 expression in NSCLC based on mass spectrometry data, explored its indirect interaction with $\mathrm{PI} 3 \mathrm{~K}$ and predicted their targeting microRNAs in common. We have also explored the potential role of miR-30 family in the modulation of PI3K-SIAH2 interaction in NSCLC.

Keywords: PI3K, SIAH2, non-small cell lung cancer, theranostics, microRNA

\section{INTRODUCTION}

Every year, about 1.8 million new lung cancer cases are diagnosed, claiming 1.6 million deaths globally. Non-small cell lung cancer (NSCLC) is a major form of lung cancer, comprising approximately $84 \%$ of all lung cancers. Although many treatment options, including targeted therapy, are available, the survival rate is still very low because of ineffective treatment outcome and acquired drug resistance. In Hong Kong, the survival rate of NSCLC is about $15 \%$ only (Ho, 2011). Although cigarette smoking has predominant association with lung cancer, non-smokers constitute a substantial minority of lung cancer patients. In the USA, 17,000-26,000 annual deaths of non-smokers from lung cancer account for the seventh leading cause of cancer mortality (Rivera and Wakelee, 2016). With the exposure to passive smoke and cooking fumes, non-smoking women represent an under-studied subset of NSCLC patients (Shen et al., 1998).

The current therapeutic approaches mainly target the molecular mechanisms of tumor cell growth caused by the aberrant activity of epidermal growth factor receptor (EGFR) (Camp et al., 2005). EGFR tyrosine kinase inhibitors (TKIs) are small molecules designed to bind to the tyrosine kinase domain of EGFR so that its phosphorylation and the subsequent receptor activation 
and signal transduction can be inhibited. However, the cancer progression remains because of the acquired resistance to EGFR TKIs (Yu et al., 2013). To date, no effective therapy is available for NSCLC patients with such resistance. Multiple molecular mechanisms of resistance have been explored, such as the activation of alternative receptor tyrosine kinases (RTKs) that bypass the EGFR signaling pathway, and the secondary mutations in EGFR T790M (Camp et al., 2005; Zhang et al., 2012). The resistance to anti-EGFR therapy may be caused by the activation of other RTKs, such as hepatocyte growth factor receptor (c-MET), Ron (a protein tyrosine kinase related to c-MET), platelet-derived growth factor receptor (PDGFR), and insulin-like growth factor receptor-1 (IGF-1R), which share the same downstream signaling cascade with EGFR pathway (Camp et al., 2005). The other activated RTKs are able to substitute the function of EGFR by activating overlapping signal transduction. The downstream molecule phosphoinositide-3-kinase (PI3K) shared by both EGFR and IGF-1R is associated with the EGFR TKIs resistance (Huang and $\mathrm{Fu}, 2015)$.

\section{PI3K Signaling Cascade Coupled with RTKs}

Upon ligand binding, RTKs dimerize and phosphorylate each other. The adaptor proteins recruit the p 85 regulatory subunit of Class IA PI3K heterodimer, which is expressed by PIK3R1, PIK3R2, PIK3R3, p85 $\beta$, and $\mathrm{p} 55 \gamma$, to the phosphorylated cytosolic RTK domains. The recruitment of p85 subunit results in a conformational change in the PI3K heterodimer that activates its p110 catalytic subunit, phosphorylates phosphatidylinositol 4,5-bisphosphate (PIP2) and converts PIP2 to phosphatidylinositol $(3,4,5)$-trisphosphate (PIP3). Protein kinase B (AKT) is then recruited by PIP3 to the cell membrane where the conserved serine residue of AKT is phosphorylated by TORC2. The phosphorylated AKT interact with a number of proteins participating in cell survival and metabolism (Manning et al., 2002).

Casitas B-lineage lymphoma (CBL) and PI3K are both downstream proteins of EGFR. CBL is an E3 ubiquitin ligase consisting of tyrosine kinase binding (TKB), RING finger domain and ubiquitin-associated domains. PI3K-phosphorylated protein activates $\mathrm{TKB}$ domain at the N-terminal of $\mathrm{CBL}$ and stimulates its ubiquitination activity. With response to this signal, RING finger domain recruits an E2 ubiquitinconjugating enzyme, such as UBE2D2, and ubiquitin-associated domain transfers ubiquitin from the enzyme to targeted substrates for degradation. Such process may promote the ubiquitination of RTK by degradation and attenuates RTK signal transduction.

\section{SIAH2 in EGFR Pathway}

The seven in absentia homolog 2 (SIAH2) is another E3 ubiquitin ligase that also mediates ubiquitination and subsequent proteasomal degradation of target proteins. In addition to $\mathrm{AKT}$ and $\mathrm{PI} 3 \mathrm{~K}, \mathrm{p} 38$ mitogen-activated protein kinase (MAPK) are the downstream proteins of EGFR pathway. Major phosphorylation sites of SIAH2, S29, and T24, contains the SQ/TQ amino acid sequence motif, which is conserved for p38 MAPK phosphorylation. Hypoxia is a condition that promotes the activities of both SIAH2 and p38 MAPK (Nakayama et al., 2009). Under hypoxic condition, p38 MAPK actively phosphorylates and stabilizes SIAH2 in cytoplasm (Emerling et al., 2005; Khurana et al., 2006). However, the role of SIAH2 in NSCLC remains unclear.

\section{Post-transcriptional Regulation by MicroRNAs}

MicroRNAs (miRNAs) represent a class of endogenous and non-coding RNAs whose mature forms are short fragments of 21-25 nucleotides long. To post-transcriptionally regulate the expression of their target genes, miRNAs bind to the miRNA response elements (MREs) of their messenger RNAs (mRNAs). MREs are the sequence motifs in $3^{\prime}$-untranslated region $\left(3^{\prime}-\mathrm{UTR}\right)$ of mRNAs complementary to the targeting miRNAs. MiRNAs are not only able to regulate the expression of the target oncogenes or tumor suppressor genes in human tumors, but also to alter the acquired drug resistance and tumor progression so that the treatment response to targeted therapy is improved particularly in NSCLC (Medina and Slack, 2008; Weiss et al., 2008; Han et al., 2015; Yu and Cho, 2015; Sin et al., 2016). It was reported that the gefitinibinduced apoptosis and epithelial-mesenchymal transition of NSCLC cells were affected by miR-30b and miR-30c through the inhibition of some oncogenes, such as sarcoma viral oncogene homolog (SRC) (Skog et al., 2008). As a miRNA able to target multiple genes, it is interesting to examine whether miR-30 family plays a theranostic role in regulating PI3K and SIAH2 concurrently and mediating the potential PI3K-SIAH2 interaction in NSCLC.

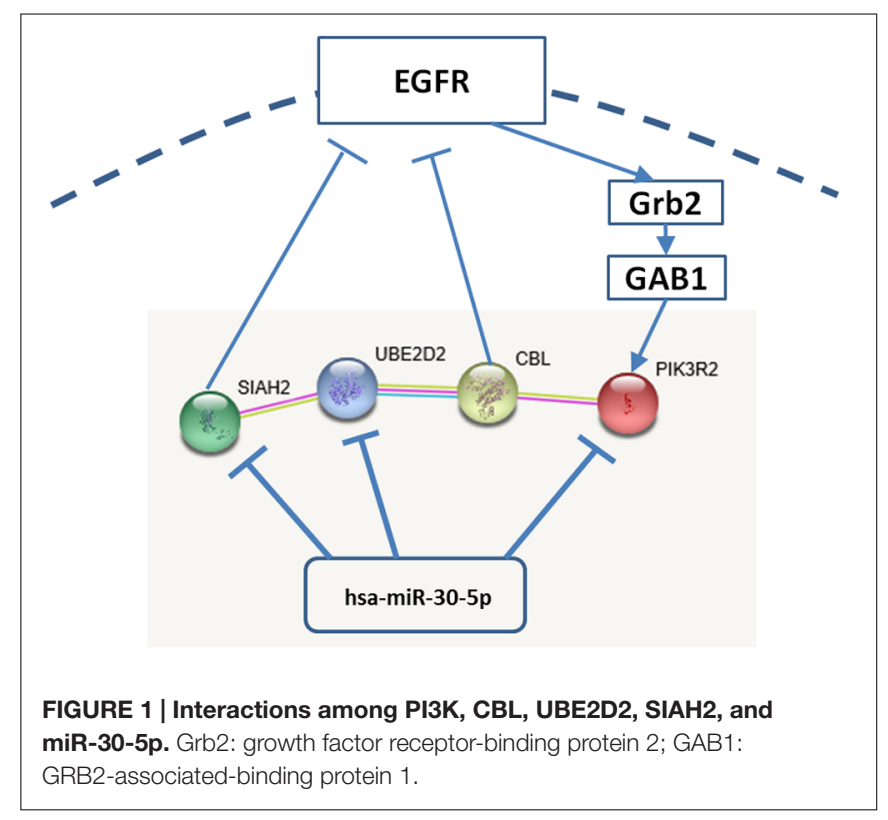




\section{MATERIALS AND METHODS}

\section{Proteomic Analysis of Blood Samples}

The plasma samples of this study were archived specimens. Among 105 patients randomly selected from pretreatment patients of Queen Elizabeth Hospital in Hong Kong, plasma samples were collected from 60 non-cancer patients and 45 adenocarcinoma patients. The non-cancer group includes 30 lung disease patients without known neoplastic tumor and 30 healthy volunteers free of any known acute or chronic illness. The adenocarcinoma group consists of 45 female nonsmokers (Eskandar et al., 2015). The plasma samples was fractionated and then profiled with the surface-enhanced laser desorption/ionization time-offlight mass spectrometer (SELDI-TOF-MS), we analyzed the array on a Proteinchip PCS4000 Reader (Ciphergen Biosystems) with acquisition up to $200 \mathrm{kDa}$ and generated the $\mathrm{m} / \mathrm{z}$ spectra by averaging a total of 338 laser shots at an intensity of 195 . The proteomic profile data from fractionation with $\mathrm{pH} 9$ with cationic $\mathrm{CM} 10$ chip was used in this study.

\section{Peptide Prediction and Differential Expression Analysis}

The molecular mass of SIAH2 is $34.615 \mathrm{kDa}$. It was predicted by Mascot MS/MS Ions Search (Matrix Science ${ }^{1}$ ) that a peptide with mass of 34461.667 Da could constitute 2-324 residues of SIAH2 and correspond to the $\mathrm{m} / z$ ratio $17230.8335 \mathrm{Da}$ in the generated mass spectra. At this $\mathrm{m} / \mathrm{z}$ ratio, we compared the intensity values of the adenocarcinoma group with that of the non-cancer group using independent sample $t$-test or its non-parameter counterpart depending on the normality of data. Differential expression of SIAH2 was indicated by $p<0.05$ in the statistical test.

\section{Protein-Protein Interaction Search}

We used Search Tool for Recurring Instances of Neighbouring Genes (STRING) to identify the potential interactions among PI3K, CBL, UBE2D2, and SIAH2. STRING hosts the information and corresponding evidence support of known and predicted protein-protein interactions in database and serves query functions and result visualizations. The interactions covered by STRING are not only experimentally-proved direct physical

${ }^{1} \mathrm{http}: / /$ www.matrixscience.com/ associations but also phylogenetic, genomic, or functional indirect associations. Evidence of identified interactions is supported by other primary databases, such as General Repository for Interaction Datasets (GRID). As of September 2016, STRING database includes the interaction information about 9,643,763 proteins from 2,031 organisms (Franceschini et al., 2013).

\section{MiRNA Target Prediction}

TargetScan was used to predict the miRNAs that concurrently regulate $\mathrm{PI} 3 \mathrm{~K}, \mathrm{CBL}, \mathrm{UBE} 2 \mathrm{D} 2$, and SIAH2. In the prediction process, TargetScan searched for the miRNAs whose seed regions match the conserved 8-mer, 7-mer, and 6-mer sites of the target genes' mRNAs (Lewis et al., 2005). We did not limit the broadly conserved, conserved, and poorly conserved but confidently annotated microRNA families and included no other microRNA annotations in our search.

\section{RESULTS}

For testing the normality of data, Shapiro-Wilk test was performed to SELDI-TOF-MS intensity data of both noncancer and adenocarcinoma groups. Normality cannot be assumed in non-cancer group $(p<0.0001)$ and adenocarcinoma group $(p=0.001)$. So, the difference between two groups was examined using non-parametric test, independent samples Mann-Whitney $U$ test. It was found that the intensities of $m / z=17230.8335 \mathrm{Da}$ were significantly different between two groups $(p=0.002$, median in noncancer $=0.1898$, median in adenocarcinoma $=0.3075$, see Supplementary Materials). This implies that the protein expression of SIAH2 is significantly upregulated in adenocarcinoma.

Search Tool for Recurring Instances of Neighbouring Genes predicted the interactions among PI3K, CBL, UBE2D2, and SIAH2 (Figure 1). All the interactions were supported by experimental results and co-mentioning in literature. The CBL-UBE2D2 interaction was further supported by the pathways curated from third-party databases, such as Kyoto Encyclopedia of Genes and Genomes (KEGG). Datasets relevant to these interactions are shown in Table 1. Although there was no evidence showing that PIK3R2 directly interact with SIAH2, TargetScan predicted that miR-30-5p family concurrently targets

TABLE 1 | Protein-protein interactions detected by assays and the corresponding hosting databases.

\begin{tabular}{|c|c|c|c|}
\hline Protein 1 & Protein 2 & Database & Detected by \\
\hline PIK3R2 & $\mathrm{CBL}$ & Intact & $\begin{array}{l}\text { Anti tag coimmunoprecipitation assay (psi-mi: "Ml:0007"), tandem affinity purification assay } \\
\text { (psi-mi: "Ml:0676"), anti bait coimmunoprecipitation assay (psi-mi: "Ml:0006") }\end{array}$ \\
\hline PIK3R2 & $\mathrm{CBL}$ & Intact, pride & Cross-linking assay (psi-mi: “Ml:0030”) \\
\hline PIK3R2 & $\mathrm{CBL}$ & GRID & Affinity capture-western assay, affinity capture-MS assay \\
\hline $\mathrm{CBL}$ & UBE2D2 & dip & Surface plasmon resonance and X-ray crystallography assays \\
\hline CBL & UBE2D2 & GRID & Reconstituted complex assay \\
\hline UBE2D2 & $\mathrm{SIAH} 2$ & GRID & Reconstituted complex assay \\
\hline
\end{tabular}


them. The involved members of miR-30-5p family include miR30a-5p, miR-30b-5p, miR-30c-5p, miR-30d-5p, and miR-30e$5 \mathrm{p}$. The type of pairing site between PIK3R2 and miR-30-5p family members is $8 \mathrm{mer}$ and the target site is conserved among mammals. The type of pairing site between SIAH2 and miR-30$5 \mathrm{p}$ family members is 7 mer-A 1 and the target site is conserved among a number of vertebrates. It is interesting to note that miR-30-5p family also targets UBE2D2.

\section{DISCUSSION AND CONCLUSION}

This work investigated the roles of PIK3R2, CBL, UBE2D2, SIAH2, and miR-30-5p family that may be related to the resistance to anti-EGFR therapy. Among these factors, SIAH2 has not been studied sufficiently and its role in cancer treatment remains unclear. In the comparison between plasma samples of independent groups, we showed the significant upregulation of SIAH2 protein in NSCLC. Moreno et al. (2015) obtained similar result but the SIAH2 expression in lung cancer was compared with that in adjacent normal tissues. Sun et al. (2016) found that SIAH2 was overexpressed in human breast cancer tissues. Our finding suggests that SIAH2 may be a novel theranostic target in the circulation of NSCLC patients.

Protein-protein interaction database search revealed the experimental proofs using the reconstituted complex assays that the E2 ubiquitin-conjugating enzyme, UBE2D2, interacts with both CBL and SIAH2, E3 ubiquitin ligases. CBL ubiquitylates active RTKs for degradation and attenuates directly the receptor signaling, including that mediated by EGFR (Joazeiro et al., 1999). The potential theranostic role of CBL has been shown in Klapper et al. (2000) that some antibodies may enhance directing HER-2 to CBL-regulated proteolysis. Similar to CBL, SIAH2 ubiquitylates protein for degradation. However, there is no evidence at the moment showing that the ubiquitination of EGFR is related to SIAH2. On the other hand, it was shown in Szargel et al. (2009) that SIAH2 is responsible for the ubiquitination of alpha-synuclein and synphilin-1 in Lewy bodies of PD patients. The only association with EGFR pathway is that both SIAH2 and MAPK are sensitive to hypoxia and MAPK, a downstream protein of EGFR can activate SIAH2 by phosphorylation (Nakayama et al., 2009).

TargetScan predicted that mRNAs of PIK3R2, UBE2D2, and SIAH 2 are concurrently targeted by miRNA-30-5p family. These three RNA transcripts sharing the same conserved MREs are called competing endogenous RNA (ceRNA). CeRNAs interact indirectly by competing for their common targeting miRNAs in cells. The up-regulation of a ceRNA could indirectly promote the expression of the other ceRNAs (Marques et al., 2011). The molecular mechanism, through which the ceRNAs are coregulated, paves the undiscovered cascades in the signaling pathway. Such co-regulation can be detected by the co-expression of ceRNAs. It was shown that PTEN and its putative ceRNAs are co-expressed in prostate cancer and glioblastoma (Tay et al.,
2011). The ceRNA crosstalk is susceptible to the miRNA:target concentration ratio and, in other words, mediated by the targeting miRNAs (Bosson et al., 2014).

Among the miRNA-30-5p family members, microRNA30 a expression was found negatively associated with the malignant potential of various NSCLC cell lines, positively with the expression of E-cadherin, which is an epithelial marker, and negatively with the expression of $\mathrm{N}$-cadherin, which is a mesenchymal marker. In other words, the epithelialto-mesenchymal transition could be inhibited by miR-30a (Kumarswamy et al., 2012). Based on the above evidence, interaction of PIK3R2-SIAH2 ceRNAs and its mediator, miRNA30-5p family, can be considered as a potential treatment target and theranostic panel in NSCLC, particularly adenocarcinoma. The treatment effect can be examined by intravenous injection of Lipofectamine reagents of miRNA-30-5p inhibitor/mimic in xenograft model and clinical trials.

\section{ETHICS STATEMENT}

The study was exempt from ethics approval because only the archived plasma samples were analyzed. No prospective study was performed.

\section{AUTHOR CONTRIBUTIONS}

LC wrote this paper, provided the research idea and performed the analysis of this work. FW advised about the association of microRNA-30 family with non-small cell lung cancer and the bioinformatics analysis. LW advised about the bioinformatics analysis. FM advised about the functional role of microRNA30 family in non-small cell lung cancer. SW advised about the molecular and genomics perspective for theranostic markers in non-small cell lung cancer. JA advised about the clinical perspective for theranostic markers in non-small cell lung cancer. SY advised about the cellular self-homeostasis under stress, particularly hypoxic response. WC provided the proteomics data and advised about the clinical diagnostic need and the bioinformatics analysis.

\section{ACKNOWLEDGMENT}

This work was supported by the Health and Medical Research Fund (HMRF, Project No. 02131026).

\section{SUPPLEMENTARY MATERIAL}

The Supplementary Material for this article can be found online at: http://journal.frontiersin.org/article/10.3389/fgene. 2017.00008/full\#supplementary-material

TABLE S1 | Intensities of $\mathrm{m} / \mathrm{z}=17230.8335 \mathrm{Da}$ across 105 blood samples. 


\section{REFERENCES}

Bosson, A. D., Zamudio, J. R., and Sharp, P. A. (2014). Endogenous miRNA and target concentrations determine susceptibility to potential ceRNA competition. Mol. Cell 56, 347-359. doi: 10.1016/j.molcel.2014.09.018

Camp, E. R., Summy, J., Bauer, T. W., Liu, W., Gallick, G. E., Ellis, L. M., et al. (2005). Molecular mechanisms of resistance to therapies targeting the epidermal growth factor receptor. Clin. Cancer Res. 11, 397-405.

Emerling, B. M., Platanias, L. C., Black, E., Nebreda, A. R., Davis, R. J., and Chandel, N. S. (2005). Mitochondrial reactive oxygen species activation of p38 mitogenactivated protein kinase is required for hypoxia signaling. Mol. Cell Biol. 25, 4853-4862. doi: 10.1128/MCB.25.12.4853-4862.2005

Eskandar, A., Ahmed, A., Daughtey, M., Kenderian, S., Mahdi, F., and Khan, A. (2015). Racial and sex differences in presentation and outcomes of small cell lung cancer in the United States: 1973 to 2010. Chest 147, e164-e165.

Franceschini, A., Szklarczyk, D., Frankild, S., Kuhn, M., Simonovic, M., Roth, A., et al. (2013). STRING v9.1: protein-protein interaction networks, with increased coverage and integration. Nucleic Acids Res. 41, D808-D815. doi: $10.1093 / \mathrm{nar} / \mathrm{gks} 1094$

Han, F., He, J., Li, F., Yang, J., Wei, J., Cho, W. C., et al. (2015). Emerging roles of microRNAs in EGFR-targeted therapies for lung cancer. Biomed. Res. Int. 2015, 672759. doi: $10.1155 / 2015 / 672759$

Ho, J. C. (2011). Targeted therapy for non-small cell lung cancer. Med. Bull. 16, 19-21.

Huang, L., and Fu, L. (2015). Mechanisms of resistance to EGFR tyrosine kinase inhibitors. Acta Pharm. Sin. B 5, 390-401. doi: 10.1016/j.apsb.2015.07.001

Joazeiro, C. A., Wing, S. S., Huang, H., Leverson, J. D., Hunter, T., and Liu, Y. C. (1999). The tyrosine kinase negative regulator c-Cbl as a RING-type, E2-dependent ubiquitin-protein ligase. Science 286, 309-312.

Khurana, A., Nakayama, K., Williams, S., Davis, R. J., Mustelin, T., and Ronai, Z. (2006). Regulation of the ring finger E3 ligase Siah2 by p38 MAPK. J. Biol. Chem. 281, 35316-35326. doi: 10.1074/jbc.M606568200

Klapper, L. N., Waterman, H., Sela, M., and Yarden, Y. (2000). Tumor-inhibitory antibodies to HER-2/ErbB-2 may act by recruiting $\mathrm{c}-\mathrm{Cbl}$ and enhancing ubiquitination of HER-2. Cancer Res. 60, 3384-3388.

Kumarswamy, R., Mudduluru, G., Ceppi, P., Muppala, S., Kozlowski, M., Niklinski, J., et al. (2012). MicroRNA-30a inhibits epithelial-to-mesenchymal transition by targeting Snail and is downregulated in non-small cell lung cancer. Int. J. Cancer 130, 2044-2053. doi: 10.1002/ijc.26218

Lewis, B. P., Burge, C. B., and Bartel, D. P. (2005). Conserved seed pairing, often flanked by adenosines, indicates that thousands of human genes are microRNA targets. Cell 120, 15-20. doi: 10.1016/j.cell.2004.12.035

Manning, B. D., Tee, A. R., Logsdon, M. N., Blenis, J., and Cantley, L. C. (2002). Identification of the tuberous sclerosis complex-2 tumor suppressor gene product tuberin as a target of the phosphoinositide 3-kinase/Akt pathway. Mol. Cell 10, 151-162. doi: 10.1016/S1097-2765(02)00568-3

Marques, A. C., Tan, J., and Ponting, C. P. (2011). Wrangling for microRNAs provokes much crosstalk. Genome Biol. 12:132. doi: 10.1186/gb-2011-1211-132

Medina, P. P., and Slack, F. J. (2008). MicroRNAs and cancer: an overview. Cell Cycle 7, 2485-2492. doi: 10.4161/cc.7.16.6453

Moreno, P., Lara-Chica, M., Soler-Torronteras, R., Caro, T., Medina, M., Álvarez, A., et al. (2015). The expression of the ubiquitin ligase SIAH2 (seven in absentia homolog 2) is increased in human lung cancer. PLOS ONE 10:e0143376. doi: 10.1371/journal.pone.0143376
Nakayama, K., Qi, J., and Ronai, Z. (2009). The ubiquitin ligase Siah2 and the hypoxia response. Mol. Cancer Res. 7, 443-451. doi: 10.1158/1541-7786.MCR08-0458

Rivera, G., and Wakelee, H. A. (2016). Resistance to therapy. Cancer Treat. Res. 170, 183-202. doi: 10.1007/978-3-319-40389-2_9

Shen, X. B., Wang, G. X., and Zhou, B. S. (1998). Relation of exposure to environmental tobacco smoke and pulmonary adenocarcinoma in non-smoking women: a case control study in Nanjing. Oncol. Rep. 5, 1221-1223.

Sin, T. K., Wang, F., Meng, F., Wong, S. C., Cho, W. C., Siu, P. M., et al. (2016). Implications of MicroRNAs in the treatment of gefitinib-resistant non-small cell lung cancer. Int. J. Mol. Sci. 17:237. doi: 10.3390/ijms17020237

Skog, J., Würdinger, T., van, Rijn S, Meijer, D. H., Gainche, L., Sena-Esteves, M., et al. (2008). Glioblastoma microvesicles transport RNA and proteins that promote tumour growth and provide diagnostic biomarkers. Nat. Cell Biol. 10, 1470-1476. doi: 10.1038/ncb1800

Sun, J., Zhang, X., Han, Y., Zhen, J., Meng, Y., Song, M., et al. (2016). Overexpression of seven in absentia homolog 2 protein in human breast cancer tissues is associated with the promotion of tumor cell malignant behavior in in vitro. Oncol. Rep. 36, 1301-1312. doi: 10.3892/or.2016.4976

Szargel, R., Rott, R., Eyal, A., Haskin, J., Shani, V., Balan, L., et al. (2009). Synphilin$1 \mathrm{~A}$ inhibits seven in absentia homolog (SIAH) and modulates alpha-synuclein monoubiquitylation and inclusion formation. J. Biol. Chem. 284, 11706-11716. doi: 10.1074/jbc.M805990200

Tay, Y., Kats, L., Salmena, L., Weiss, D., Tan, S. M., Ala, U., et al. (2011). Coding-independent regulation of the tumor suppressor PTEN by competing endogenous mRNAs. Cell 147, 344-357. doi: 10.1016/j.cell.2011. 09.029

Weiss, G. J., Bemis, L. T., Nakajima, E., Sugita, M., Birks, D. K., Robinson, W. A., et al. (2008). EGFR regulation by microRNA in lung cancer: correlation with clinical response and survival to gefitinib and EGFR expression in cell lines. Ann. Oncol. 19, 1053-1059. doi: 10.1093/annonc/mdn006

Yu, H. A., Sima, C. S., Huang, J., Solomon, S. B., Rimner, A., Paik, P., et al. (2013). Local therapy with continued EGFR tyrosine kinase inhibitor therapy as a treatment strategy in EGFR mutant advanced lung cancers that have developed acquired resistance to EGFR tyrosine kinase inhibitors. J. Thorac. Oncol. 8, 346-351. doi: 10.1097/JTO.0b013e31827e1f83

Yu, H. W., and Cho, W. C. (2015). The emerging role of miRNAs in combined cancer therapy. Expert Opin. Biol. Ther. 15, 923-925. doi: 10.1517/14712598. 2015.1030390

Zhang, Z., Lee, J. C., Lin, L., Olivas, V., Au, V., LaFramboise, T., et al. (2012). Activation of the AXL kinase causes resistance to EGFR-targeted therapy in lung cancer. Nat. Genet. 44, 852-860. doi: 10.1038/ng.2330

Conflict of Interest Statement: The authors declare that the research was conducted in the absence of any commercial or financial relationships that could be construed as a potential conflict of interest.

Copyright (c) 2017 Chan, Wang, Meng, Wang, Wong, Au, Yang and Cho. This is an open-access article distributed under the terms of the Creative Commons Attribution License (CC BY). The use, distribution or reproduction in other forums is permitted, provided the original author(s) or licensor are credited and that the original publication in this journal is cited, in accordance with accepted academic practice. No use, distribution or reproduction is permitted which does not comply with these terms. 